\title{
Monte Carlo calculation of high-energy heavy-ion interactions
}

\author{
J. Cugnon* \\ W. K. Kellogg Radiation Laboratory, California Institute of Technology, Pasadena, California 91125 \\ (Received 4 February 1980)
}

\begin{abstract}
A relativistic Monte Carlo model for high-energy heavy-ion collisions is presented. The interaction process is described as a sequence of classical, binary, on-shell baryon-baryon collisions. Pion production is taken into account by allowing $\Delta$ resonance formation. The latter are given a definite mass and a lifetime larger than the collision time. They are, however, allowed to scatter or disappear by collisions with the nucleons. The results of the calculations are compared with experimental results for the inclusive proton and pion cross sections and two-proton correlations in the ${ }^{12} \mathrm{C}+{ }^{12} \mathrm{C},{ }^{20} \mathrm{Ne}+\mathrm{NaF}$, and ${ }^{40} \mathrm{Ar}+\mathrm{KCl}$ systems at $800 \mathrm{MeV}$ per nucleon. The predictions of the model agree fairly well with the experimental measurements, except for the low-energy pion cross section, which is underestimated. The relation of this feature to the zero-width mass spectrum of the $\Delta$ resonances is briefly discussed. The model is used to separate the direct knockout and the thermal contributions to the proton inclusive cross section. Attention is drawn to the fact that these two contributions are not unambiguously defined.
\end{abstract}

$\left.\begin{array}{c}\text { NUCLEAR REACTIONS High-energy heavy-ion collisions; Monte Carlo simu- } \\ \text { lation; calculated proton and pion spectra for }{ }^{12} \mathrm{C}+{ }^{12} \mathrm{C},{ }^{20} \mathrm{Ne}+\mathrm{NaF} \text {, and }{ }^{40} \mathrm{Ar} \\ +\mathrm{KCl} \text { at } E / A=800 \mathrm{MeV}\end{array}\right]$

\section{INTRODUCTION}

The recent availability of accelerators capable of providing heavy ion beams with a kinetic energy of a few hundreds of $\mathrm{MeV}$ to a few $\mathrm{GeV}$ per nucleon has opened a new chapter of nuclear physics, where the atomic nuclei are studied under quite unusual conditions. It was believed that such high energy reactions possibly were the site of exotic phenomena such as shock wave, ${ }^{1}$ pion condensation, ${ }^{2}$ or density isomers. ${ }^{3}$ At present, the situation is rather disappointing. The evidence for these exotic phenomena (or any others) is rather weak. The detection of their occurrence is difficult for the following two reasons: (i) in some cases, at least, one does not know very well what is the signature of the exotic phenomena, and (ii) this signature, if any, is superimposed on a huge background due to "conventional" nuclear processes. It is then highly desirable to calculate this background accurately. A calculation based on a fully quantum mechanical theory, if it exists, is not to be expected in the near future. As a consequence, one has been forced to rely on models, based on suitable simplifying assumptions. Among these, the fireball ${ }^{4}$ or firestreak model, ${ }^{5}$ the hydrodynamical models, ${ }^{6,7}$ and the direct knockout model $^{8}$ have been particularly successful in calculating the main features of the inclusive proton and pion cross sections. In our opinion those models contain strong (and contradictory) hypotheses concerning the number of binary nucleonnucleon collisions occurring during the nucleusnucleus interaction process. As an alternative a certain class of models has been devised which takes into account the contribution of any number of successive nucleon-nucleon collisions; these are the classical equations of motion approach, ${ }^{9}$ the "rows on rows" model, ${ }^{10}$ and the intranuclear cascade model. ${ }^{11,12}$ All of these models constitute a substantial effort to construct an accurate picture of the interaction as a sequence of independent nucleon-nucleon collisions.

Our work is a contribution to this effort. We present the results of a Monte Carlo calculation for relativistic heavy-ion collisions. The nuclei, both target and projectile, are described initially as free Fermi gases. The projectile is boosted against the target with the appropriate velocity. The nucleons are made to scatter, by criteria explained below, according to the experimental nucleon-nucleon cross sections. In this way, we take full account of the multiple collision sequence in a classical, relativistic picture. Pion production is introduced via the formation of $\Delta$ resonances.

We distinguish between a Monte Carlo calculation and the existing intranuclear cascade models because, as we explain below, the former includes compression and decompression effects, even if these do not markedly change the observables we calculate.

In Sec. II, we describe our model in detail. In Sec. III, we present the results for the inclusive proton and pion cross sections in collisions of ${ }^{12} \mathrm{C}$ on ${ }^{12} \mathrm{C},{ }^{20} \mathrm{Ne}$ on ${ }^{20} \mathrm{Ne}$, and ${ }^{40} \mathrm{Ar}$ on ${ }^{40} \mathrm{Ar}$ at $800 \mathrm{MeV}$ per nucleon incident kinetic energy in the laboratory system. Section IV displays some results for two nucleon correlations. In Sec. V, we attempt to assess the relative importance of the knockout 
process, where nucleons escape the system after making a single collision. Section VI contains our conclusion.

\section{THE MONTE CARLO MODEL}

We list here the main features of our model and point out the basic difference between our model and a typical intranuclear cascade model. ${ }^{12}$

(1) The initial positions of the nucleons are generated randomly, both in the projectile and the target rest frames, according to a uniform spherical distribution of radius $R=1.12 A^{1 / 3} \mathrm{fm}$. For the ${ }^{12} \mathrm{C}$ nucleus, however, we take a radius parameter of $1.3 \mathrm{fm}$, a value which fits the experimental $\mathrm{rms}$ charge radius.

(2) The nucleons are given an initial momentum in the target or projectile rest frame according to a free Fermi gas law with the Fermi momentum $P_{F}=270 \mathrm{MeV} / c$.

(3) We neglect the binding energy of the nucleons in the nuclei. We prefer this prescription rather than introducing a potential well because the latter involves the unpleasant feature of nucleons colliding off their mass shell. Moreover, the coherence of the average field is most probably quickly destroyed, at least in central collisions. Binding corrections are, however, compulsory. In some cases, their gross effect is easy to predict. For example, protons escaping at large angles in the c. $m$. frame will have less kinetic energy than we predict, by an amount equal to the average potential energy, say $\sim 25 \mathrm{MeV}$. This would change our results only slightly (see Figs. 1, 2, 4, and 6). The real correction is hard to apply since it depends upon the way the average field is modified during the collision process.

The absence of a potential well in our calculation prevents our describing correctly the production of composite particles as well as the projectile fragmentation. In the cases we investigated below, composite particle production is a sizable correction at small angles only and becomes progressively negligible at large angles (see Sec. III).

Another consequence of the lack of potential wells is that the nuclei can expand, even in the absence of nucleon-nucleon collisions, just because of Fermi motion. This effect is, however, not important because of short collision times (see below).

(4) The projectile is boosted with the incident ion velocity. The positions and the momenta of the nucleons are changed by the appropriate Lorentz transformation. Relativistic kinematics are used everywhere. All the calculations reported here are performed in the target rest frame.
(5) The nucleons travel along straight line trajectories until the relative distance between two of them in their c.m. frame reaches a minimum. ${ }^{13}$ They are then made to scatter according to the scenario of point (7). Free motion is resumed until the relative distance is minimum for another pair. These are then made to scatter, and so on. The chain of binary collisions is followed up to a time where the number of nucleon-nucleon collisions per unit time becomes negligible. This happens quite abruptly as shown in Ref. 14, where the properties and the assumptions of the model are studied in detail. In all the systems considered below, it always occurs before $t=14 \mathrm{fm} / c$, where we stop the process. (The origin of the time scale corresponds to the configuration where the nuclei are touching before the collision.)

(6) The pionic degrees of freedom are taken into account by introducing the possibility of creating $\Delta$ resonances. The behavior of the $\Delta$ resonance in nuclear matter is rather poorly known, especially for nonequilibrium situations. We have chosen to introduce $\Delta$ resonances with a definite mass of $m_{\Delta}=1232 \mathrm{MeV}$, and a lifetime longer than the interaction time (they are therefore called $\Delta$ particles hereafter). The existence of $\Delta$ particles in nuclear matter with a rather long lifetime against pion emission is supported by some theoretical investigations. ${ }^{15,16}$ Moreover, it is shown in Ref. 17 that, in the context of a model similar to the present one, the $\pi^{-}$multiplicities can be reproduced only with long-lived $\Delta$ particles.

The $\Delta$ particles are, however, allowed to be destroyed or scattered by colliding with the nucleons. More precisely, the following reactions are explicitly taken into account:

$$
\begin{array}{ll}
N+N \rightarrow N+N, & N+N \rightarrow N+\Delta, \\
N+\Delta \rightarrow N+\Delta, & N+\Delta \rightarrow N+N, \\
\Delta+\Delta \rightarrow \Delta+\Delta . &
\end{array}
$$

The nucleon-nucleon cross section is taken from experiment. ${ }^{18,19}$ The other elastic cross sections are assumed to be equal to the nucleon-nucleon elastic cross section at the same c. $m$. energy. This assumption is not a drastic one, the results being fairly insensitive to the precise value of the elastic cross sections involving $\Delta$ particles, as shown in Ref. 14. The cross section for $\Delta$ formation is taken as the experimental inelastic nucleon-nucleon cross section, ${ }^{18}$ and the recombination $(N+\Delta \rightarrow N+N)$ cross section is obtained by detailed balance. At the end of the multiple collision process, the $\Delta$ particles are allowed to decay isotropically into a pion and a nucleon.

(7) When for a pair of baryons (nucleon or delta) the minimum distance at approach $(d)$ is at- 
tained, their motion is perturbed only if

$$
d \leqslant \frac{\sigma_{\text {tot }}(\sqrt{s})}{\pi}
$$

where $\sigma_{\text {tot }}(\sqrt{s})$ is the total cross section corresponding to the state of the incoming pair of baryons at the c.m. energy $\sqrt{s}$. The final state is chosen randomly according to the relative weights of the different reaction cross sections. The momenta of the outgoing baryons are generated randomly according to the angular distribution and in agreement with the energy-momentum conservation laws. Conservation of angular momentum is, however, destroyed by this procedure. For elastic scattering, the angular distribution has a slope $e^{B t}$, where $t$ is minus the square momentum transfer and $B$ is a smooth function of energy fitted to the experimental values. For $\Delta$ formation and recombination, we take an isotropic angular distribution, in view of the small kinetic energy in the production channel.

(8) We suppress soft collisions. If the c. m. kinetic energy of a pair of nucleons (not $\Delta^{\prime} s$ ) is less than $50 \mathrm{MeV}$, they do not scatter. This partly accounts for Pauli principle, since collisions between nucleons within a Fermi sphere with an equlibrium value of the Fermi momentum are partly suppressed by this prescription. We have checked in a typical example that the main features of the results are insensitive to the precise cutoff value.

(9) The isospin degrees of freedom are not taken into account explicitly. Thus we only deal with one sort of nucleon, one sort of $\Delta$, and one sort of pion. The main feature which distinguishes our calculation from intranuclear cascades is embodied in point (5). We picture the process as a succession of baryon-baryon collisions while intranuclear cascade codes rather consider interaction of the incident nucleons with a piece of matter providing them with a random mean free path (this procedure is symmetrized between target and projectile). Obviously, in our model the matter density at a given point can vary more widely. ${ }^{17}$ Moreover, the interaction between cascading particles, often neglected by intranuclear cascade codes, is automatically included in our model.

\section{INCLUSIVE CROSS SECTIONS}

Figure 1 shows the results of the calculation for the invariant proton inclusive cross section for a typical system. The measurements are those of Nagamiya et al. , ${ }^{20}$ who bombarded a $\mathrm{KCl}$ target with $800 \mathrm{MeV}$ per nucleon ${ }^{40} \mathrm{Ar}$ ions. To compare with this rather unusual system, we considered the symmetric system ${ }^{40} \mathrm{Ar}+{ }^{40} \mathrm{Ar}$. We use this name

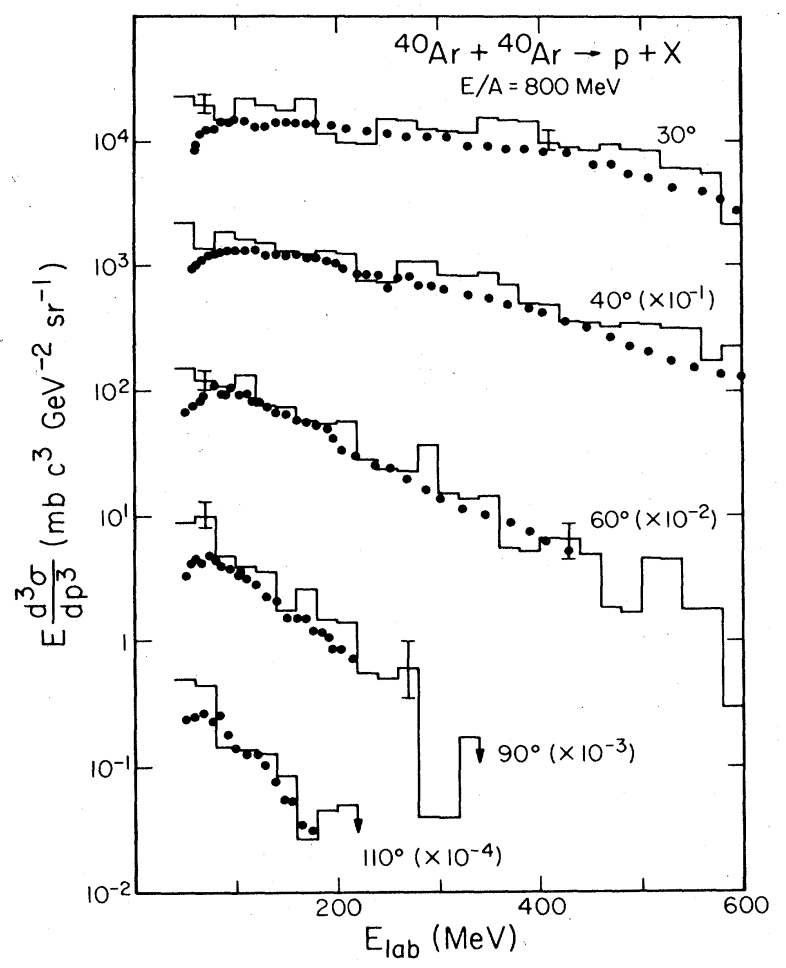

FIG. 1. Invariant proton inclusive cross section for the ${ }^{40} \mathrm{Ar}+{ }^{40} \mathrm{Ar}$ system at $800 \mathrm{MeV}$ incident kinetic energy per nucleon in the laboratory system. The cross section is plotted as a function of the kinetic energy of the emitted proton in the laboratory system. The dots are the experimental data from Ref. 20 for the ${ }^{40} \mathrm{Ar}+\mathrm{KCl}$ system. The histograms are the results of our Monte Carlo calculation. Typical error bars for the calculation are shown. A vertical arrow means that no proton is detected beyond that energy. The data are not corrected for composite particle emission. The magnitude of the correction is smaller than or comparable to the uncertainties of the calculation (see the text).

to make later identification easier, but recall that we consider one type of nucleon. The histogram represents half of the calculated nucleon inclusive cross section. The agreement with the experiment is quite good. It should, however, be kept in mind that Fig. 1 only covers a fraction of the region that can be reached by the nucleons in the transverse momentum $\left(p_{1}\right)$-rapidity $(y)$ plane. Figure 2 displays a much wider zone in this plane. To give an idea, a laboratory kinetic energy of $600 \mathrm{MeV}$ corresponds to a momentum $p_{1 \mathrm{ab}} \approx 1.2 \mathrm{GeV} / c$.

The huge peak in the calculated yield at $10^{\circ}$ is from the spectator part of the projectile, which travels as an almost undisturbed Fermi sphere. It appears at small laboratory angles and with the beam velocity. This object is counted in our calculation as a collection of individual nucleons, 


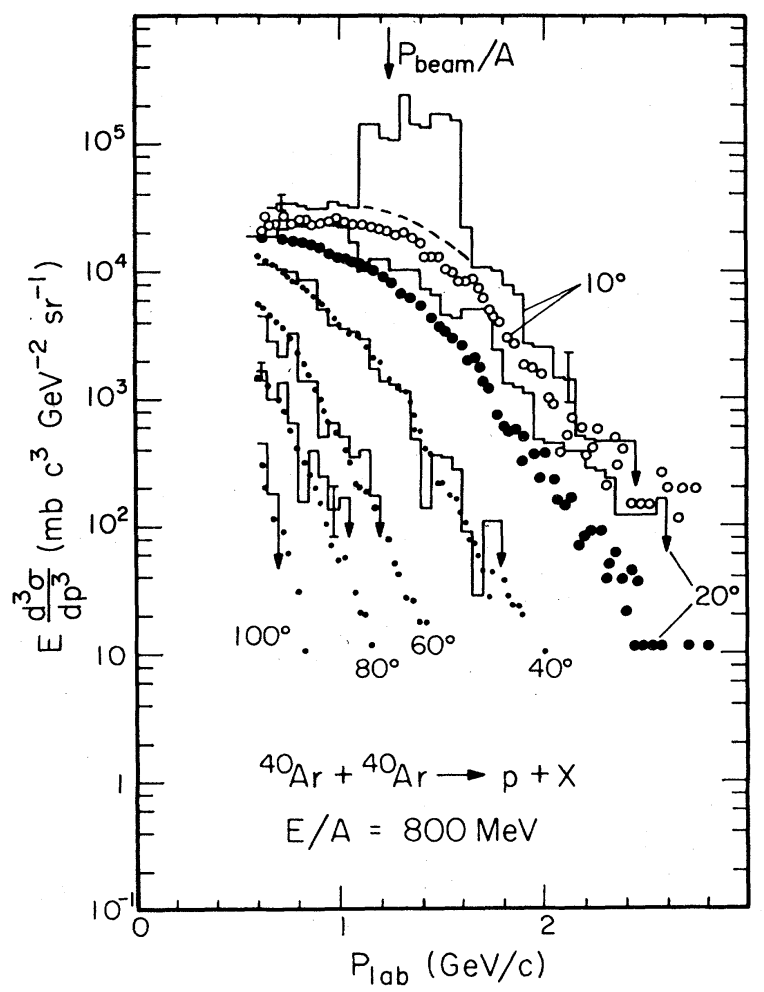

FIG. 2. Invariant proton cross section for the ${ }^{40} \mathrm{Ar}$ $+{ }^{40} \mathrm{Ar}$ system at $800 \mathrm{MeV}$ incident kinetic energy per nucleon in the laboratory system, as a function of the emitted proton momentum. The histograms are the results of our Monte Carlo calculation. Typical error bars for the calculation are shown. The vertical arrows mean that no proton is observed beyond that energy. The data (from Ref. 20) are corrected for deuteron emission (see text).

whereas in the experiment, it is observed as one or several heavy fragments. It is a straightforward consequence of our neglect of a potential well. It can be disregarded by considering the broken line, which interpolates smoothly the calculated curve over the peak region.
A similar correction has to be applied for the deuterons that are emitted by the participant part of the system. For this reason, we have compared our theoretical histograms with the experimental proton cross section, corrected for the protons contained in the deuterons. This correction is essentially the experimental deuteron cross section, scaled to account for the fact that the momentum of the proton is half the deuteron momentum. Table I shows the magnitude of this correction. It is sizable at $10^{\circ}$ and $20^{\circ}$ only. Incidentally, we note that the deuteron emission modifies Fig. 1 only slightly and actually improves the agreement a bit at $30^{\circ}$.

We did not include the correction for tritons, ${ }^{3} \mathrm{He},{ }^{4} \mathrm{He} .$. . , because we had no experimental information at our disposal for the systems we investigated. However, measurements on a similar system, ${ }^{40} \mathrm{Ar}+\mathrm{Ca}$ (at higher energy, however), ${ }^{22}$ and systematics of Ref. 23 seem to indicate that the relative importance of composite particle production increases when (i) the energy decreases, (ii) the mass of the system increases, and (iii) the observation angle decreases. From these pieces of information, one can rather safely conclude that for the cases we study here, the emission of tritons, ${ }^{3} \mathrm{He}$, and ${ }^{4} \mathrm{He}$ contribute, at most, $10 \%$ in the most favorable case, i.e., $\mathrm{Ar}+\mathrm{Ar}$ at $10^{\circ}$. Their contribution is negligible for $\theta_{1 a b}$ larger than $30^{\circ}$. We draw attention to the fact that the emission of composite particles plays a more important role in larger systems at smaller energy. ${ }^{23}$

One can see from Fig. 2 that we get fairly good agreement with the experimental measurements. There are, however, two systematic discrepancies. At small angles, the calculated cross section is a little bit too large, while at large momenta, the theoretical cross section drops off too rapidly. The first disagreement can be attributed, at least partly, to the emission of other light particles (tritons, ${ }^{3} \mathrm{He},{ }^{4} \mathrm{He}$ ) by the participant part of the

TABLE I. Average values of the scaled deuteron cross section (Ref. 21) in the ${ }^{40} \mathrm{Ar}+{ }^{40} \mathrm{Ar}$ system at $800 \mathrm{MeV}$ per particle (see text). They are given in $\mathrm{mb}^{3} \mathrm{GeV}^{-2} \mathrm{sr}^{-1}$.

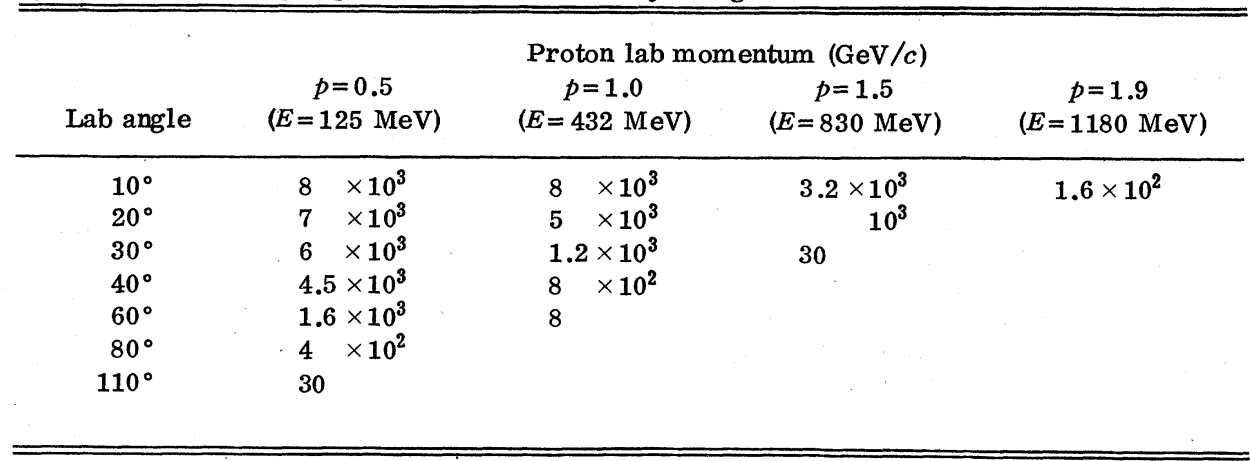


system. However, it is hard to know whether the second discrepancy is due to a lack of statistics or to a kinematical limitation of high energy nucleon production.

In Fig. 3, we have plotted the pion inclusive cross section in the same system. The heavy dots represent the negative pion cross section measurements of Ref. 20. Since we disregard isospin in the calculation, we have plotted one third of the inclusive cross section for the single sort of pions we have (the ratio of one third is consistent with $\Delta$ resonance dominance). Hence the histogram describes the $\pi^{+}$as well as the $\pi^{-}$ yield. Although we obtain the right order of magnitude, there is a substantial discrepancy between the predictions of the model and the experiment. This discrepancy reduces with increasing angle and can be characterized in this way: The average yield is a little bit too high and too few low energy pions are produced at small angles. In other words, pion production with low energy in the c. $m$. system is underestimated. This can be traced back to our simplifying assumption of a constant $\Delta$ mass. Indeed, if the $\Delta$ particles

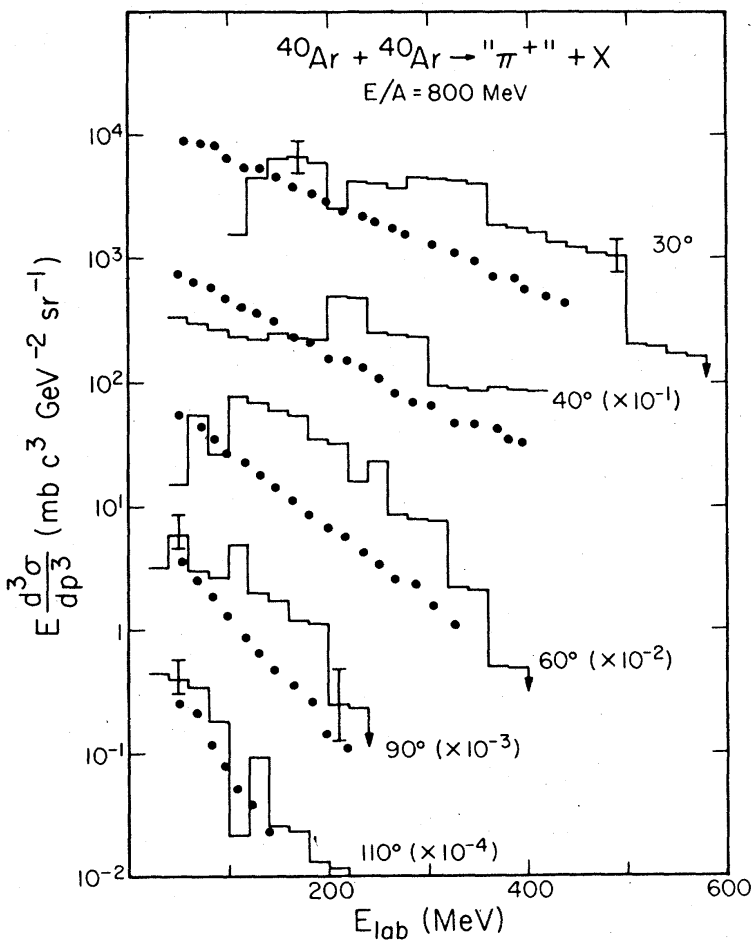

FIG. 3. Invariant pion inclusive cross section for the ${ }^{40} \mathrm{Ar}+{ }^{40} \mathrm{Ar}$ at $800 \mathrm{MeV}$ per nucleon. The dots represent the negative pion data of Ref. 20 for the ${ }^{40} \mathrm{Ar}+\mathrm{KCl}$ system. Error bars for the results of our Monte Carlo calculation (histograms) are shown. have a thermal spectrum, they will produce very few low energy pions, because a $\Delta$ particle of $1232 \mathrm{MeV}$ mass at rest decays by producing a pion with a momentum of roughly $250 \mathrm{MeV} / c$. Allowing for a wider spectrum for the $\Delta$ mass would generate a better pion spectrum at low energy.

Results for lighter systems are shown in Figs. 4 to 7. The proton inclusive cross sections are very well reproduced even at large angles. Corrections for deuteron emission are not included. They will improve the agreement at small angles. The calculated pion inclusive cross sections get closer to the experimental values when going to lighter systems, but still suffer from the same defects as in the ${ }^{40} \mathrm{Ar}+{ }^{40} \mathrm{Ar}$ system.

In Figs. 8 and 9, we present inclusive results which explore the $y-p_{\perp}$ plane in a different and complementary way. They are shown in the total c. $m$. frame. One can see, in Fig. 8, that the proton yield at $90^{\circ} \mathrm{c.m}$. is correctly reproduced

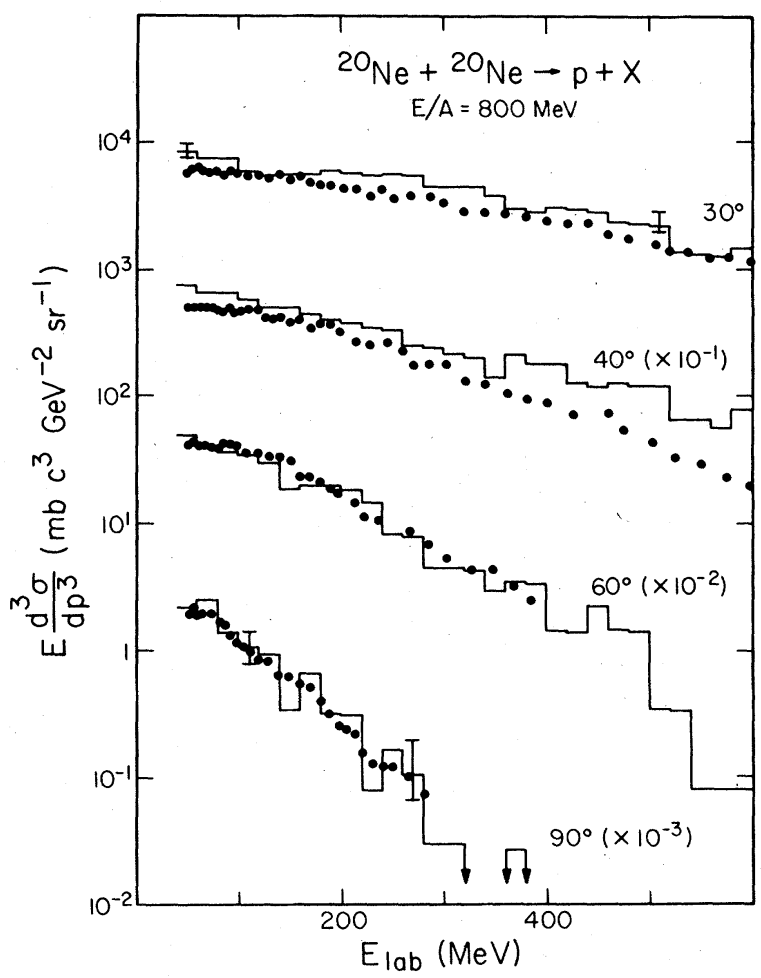

FIG. 4. ${ }^{20} \mathrm{Ne}+{ }^{20} \mathrm{Ne}$ system. Invariant proton cross section at $800 \mathrm{MeV}$ incident kinetic energy per nucleon in the laboratory system, as a function of the proton kinetic energy. The dots are the experimental data of Ref. 20 for the ${ }^{20} \mathrm{Ne}+\mathrm{NaF}$ system. The results of our calculation are shown by histogram. Typical uncertainties are displayed. The data are not corrected for composite particle emission. Corrections are expected to lie within the uncertainty of the calculation (see text). 


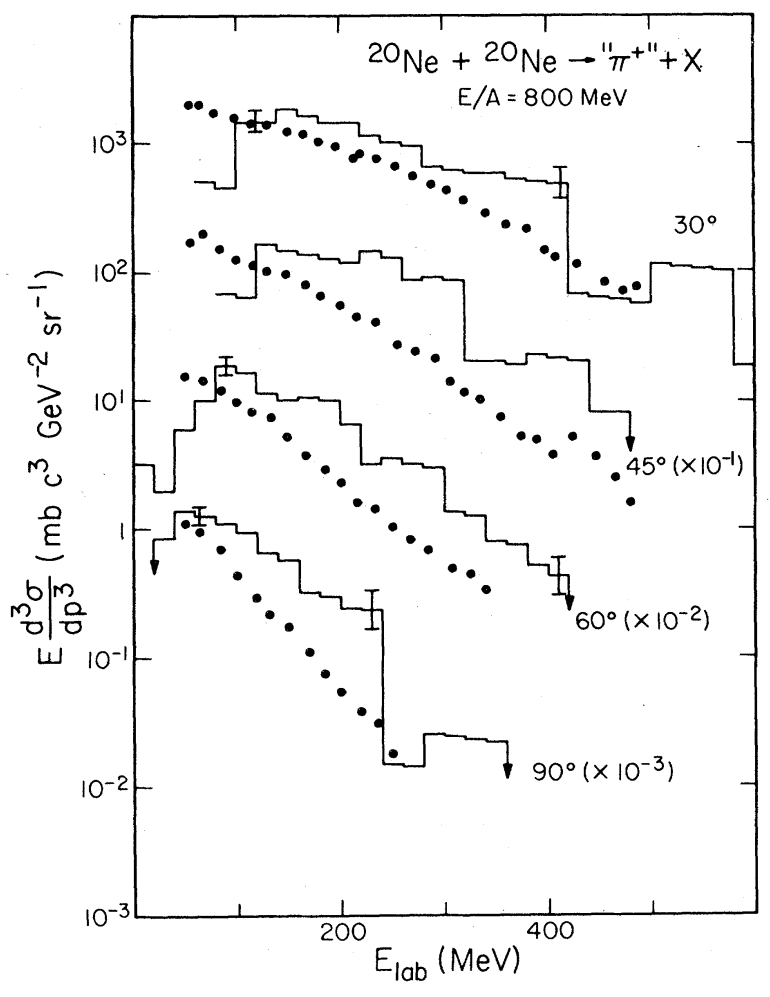

FIG. 5. Invariant pion inclusive cross sections for ${ }^{20} \mathrm{Ne}+{ }^{20} \mathrm{Ne}$ at $800 \mathrm{MeV}$ per nucleon. The dots are negative pion data of Ref. 20 for the ${ }^{20} \mathrm{Ne}+\mathrm{NaF}$ system. Error bars for the results of our Monte Carlo calculation (histograms) are shown.

even at high energy. Figure 9 shows that we obtain a good agreement for the c.m. angular distributions. The agreement deteriorates at 600 MeV kinetic energy, most probably because the statistics are poor in this region. The inclusive quantities displayed in the last two figures are often considered as possibly containing information about the reaction mechanism. We will come back to this point in Sec. V.

\section{TWO-NUCLEON CORRELATIONS}

There has been an extensive experimental effort to perform correlation measurements ${ }^{24,25}$ which are expected to shed some light on the reaction mechanism. We have calculated the two-nucleon correlation coefficient for several cases. The definition of this coefficient is given in Ref. 25. Let us, however, describe briefly the experimental configuration to make the following figures understandable. The experiment involves four counters: $T, U, R$, and $D$, the last three being positioned at a polar angle of $40^{\circ}$. The polar angle $\theta$ of $T$ can be varied. Their azimuthal angles

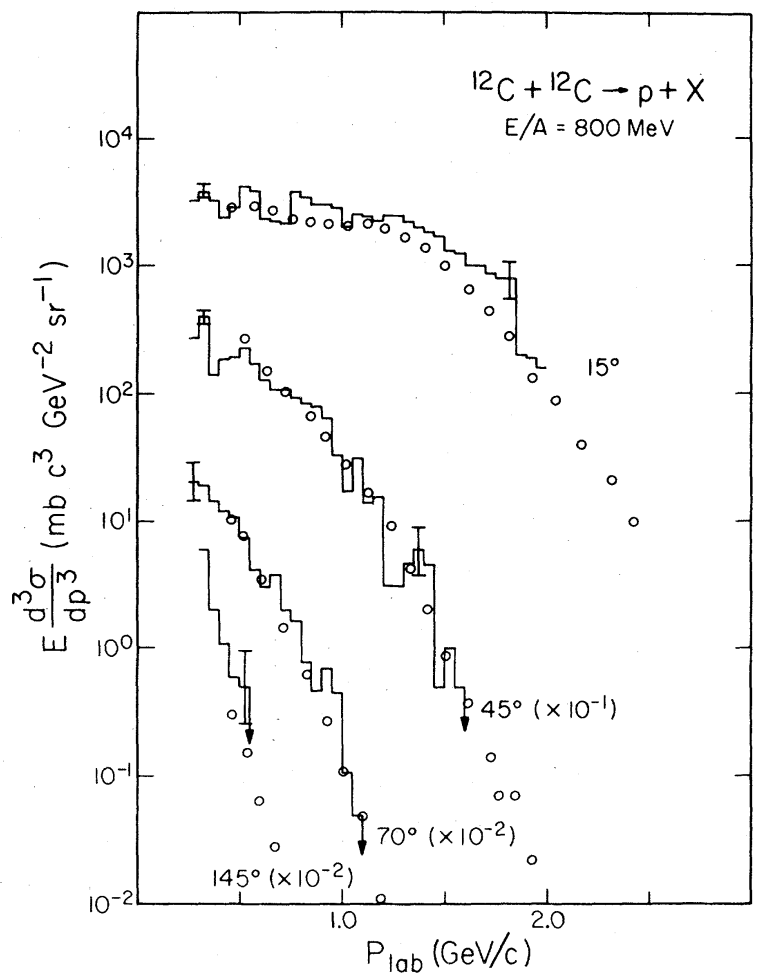

FIG. 6. Invariant proton inclusive cross section for the ${ }^{12} \mathrm{C}+{ }^{12} \mathrm{C}$ system at a beam energy of $800 \mathrm{MeV}$ per nucleon. Data (dots) are from Ref. 20. The histograms show the result of our calculation. The data are not corrected for the composite particle emission. This correction is expected to be smaller than the uncertainty of the calculation (shown by the error bars). See the text for the discussion.

$\varphi$ are such that if we assume $T$ to be at $\varphi=0$, then $U, R$, and $D$ are located at $\varphi=90^{\circ}, 180^{\circ}$, and $270^{\circ}$, respectively. The protons are counted in $U, R$, and $D$, if, and only if, a proton is detected by the counter $T$. The correlation coefficient $C(\theta)$ is essentially twice the ratio of the counting rate in $R$ to the sum of the counting rates in $U$ and $D$. The angle of $40^{\circ}$ corresponds roughly to $90^{\circ}$ in the c.m. system for the kinematics we are considering here. The quantity $C(\theta)$ is expected to have a sharp peak at $\theta=40^{\circ}$ in the case where the collision proceeds via the single knockout mechanism only. On the other hand, $C(\theta)$ will be equal to 1 , independent of angle, if the multiple collision (thermal) mechanism dominates. In Fig. 10 , we show our results at $800 \mathrm{MeV}$ per nucleon along with the experimental values. ${ }^{24}$ One can see that we reproduce these data in the average. For the ${ }^{40} \mathrm{Ar}+{ }^{40} \mathrm{Ar}$ system, however, we predict a little more correlation than is indicated by the experiment, while for the ${ }^{12} \mathrm{C}+{ }^{12} \mathrm{C}$ system, the 




FIG. 7. Invariant pion inclusive cross section for the ${ }^{12} \mathrm{C}+{ }^{12} \mathrm{C}$ system at a beam energy of $800 \mathrm{MeV}$ per nucleon. Data (dots) from Ref. 20. The histograms are the results of our calculation.

calculated peak is located at a somewhat larger angle than the experimental one. It is difficult to assign the origin of these discrepancies to a particular feature of our model. For ${ }^{20} \mathrm{Ne}+{ }^{20} \mathrm{Ne}$, we had only the $E / A=400 \mathrm{MeV}$ data at our disposal. This part of the figure is therefore only indicative, although the $E / A=800 \mathrm{MeV}$ data are not expected to be very different. ${ }^{24}$

More precise information can be gained if the counters $R, U$, and $D$ count protons, if, and only if, the telescope $T$ detects a proton of a given energy. The quantity $C$ is then a function of both $\theta$ and the energy of the particle or, equivalently, of its parallel and perpendicular momentum components $\left(p_{11}\right.$ or $\left.p_{1}\right)$ in the c.m. system. The quantity $C$ is shown in Fig. 11 for the ${ }^{12} \mathrm{C}+{ }^{12} \mathrm{C}$ system. The shaded area indicates the domain of energy for the protons detected in the $R$ counter. Once again, although the statistics are not good, we are in overall agreement with the experiment. There is, however, a slight deviation in that the ridge is not exactly positioned. The maximum value is located at too low a momentum. This situation is corroborated by Fig. 12, which displays the values of the coefficient $C\left(p_{1}, p_{\| 1}\right)$ for a laboratory angle of

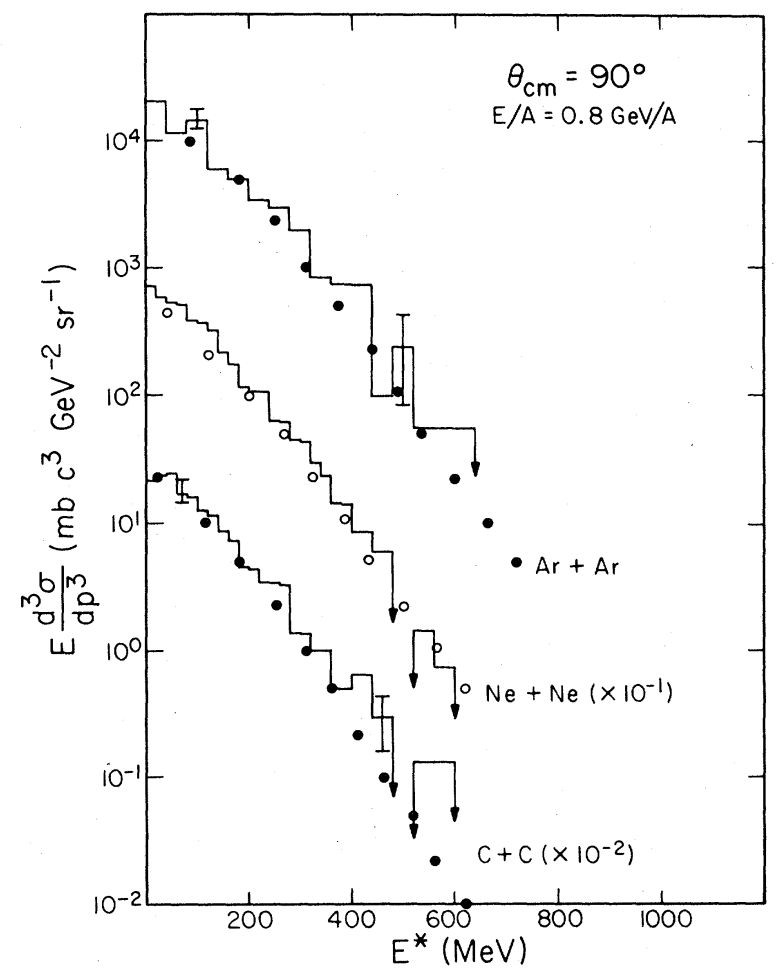

FIG. 8. Invariant inclusive proton cross section at $90^{\circ} \mathrm{c.m}$. for different systems plotted as a function of the proton kinetic energy in the c.m. frame.

$40^{\circ}$, i. e., for a cut in the $\left(p_{1}, p_{11}\right)$ plane. Once again, there is rather good agreement between theory and experiment, but the peak is too high and occurs at too low a value of $p_{\perp}$.

\section{SINGLE KNOCKOUT VERSUS MULTIPLE COLLISIONS}

The first inclusive data had been explained successfully by the fireball model, ${ }^{4}$ in which the so-called participant nucleons suffer so many collisions that they are thermalized. On the other hand, Koonin and $\mathrm{Hatch}^{8}$ have shown that the 800 $\mathrm{MeV} / A$ data can be explained by a single knockout process. In this picture, the nucleons of the projectile collide once, at most, with the target nucleons. Since that time, there has been intensive effort to try to discriminate between the two processes. Before discussing this point in connection with our results, we would like to draw attention to the fact that the two processes are not defined unambiguously. In the fireball model, the quantity which is not precisely defined is the number of participant nucleons. In the knockout model, the flexible parameter is associated with the tail of the nucleon momentum distribution. 


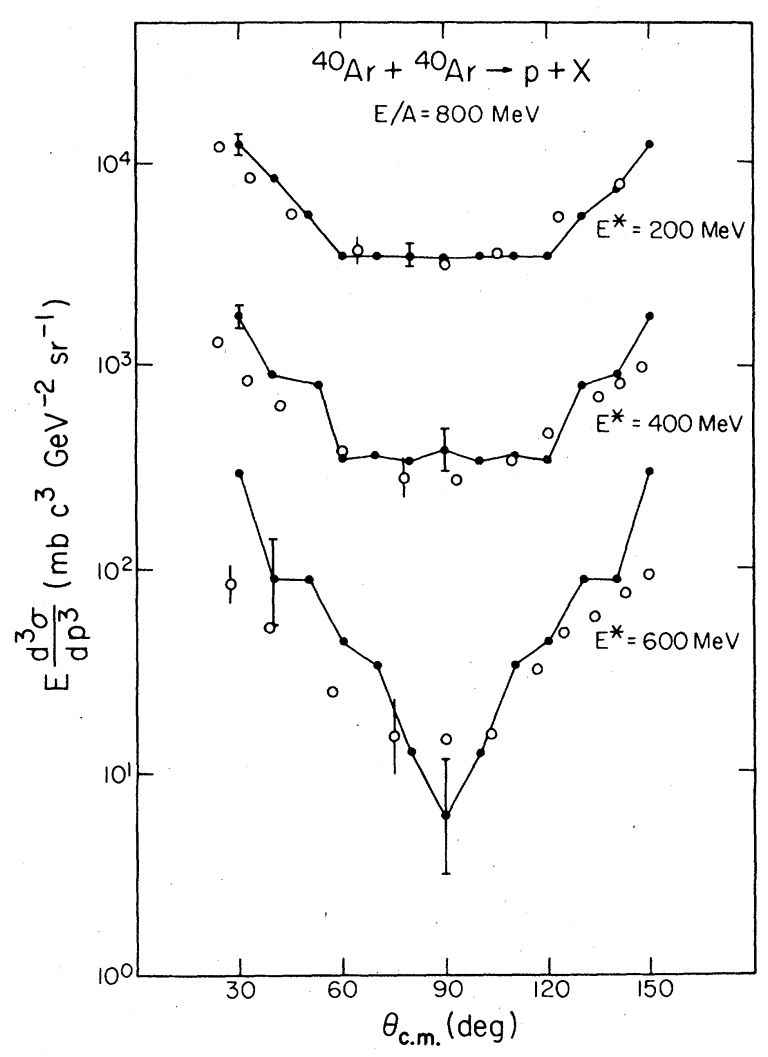

FIG. 9. Invariant inclusive proton cross section as a function of the c.m. angle for the ${ }^{40} \mathrm{Ar}+{ }^{40} \mathrm{Ar}$ system at $800 \mathrm{MeV}$ per nucleon.

The first and simple analysis would consist of comparing the predictions of both models, as they have been developed, with the experiment. In this respect, the data considered in Figs. 1 through 7 are useless, because they are reproduced equally well by almost any of the models which have been proposed. The inclusive cross section at $90^{\circ} \mathrm{c.m}$. and especially the angular distribution in the c.m. frame provided a more sensitive test. The data suggest that the physical reality lies somewhere between the two extreme models: In the fireball model, the protons are emitted isotropically, while the knockout model predicts angular distribution more forward peaked than observed experimentally. It would then be interesting to determine the relative weight of the two processes. The two-particle correlation could be considered as suitable for that purpose. They show definite departure from pure thermal process. An extended analysis of the data ${ }^{21}$ leads to the conclusion that the knockout process contributes up to $50 \%$, fairly independently of the mass of the system: Unfortunately, this analysis is slightly complicated by the existence of Fermi motion, which broadens the correlation peaks and

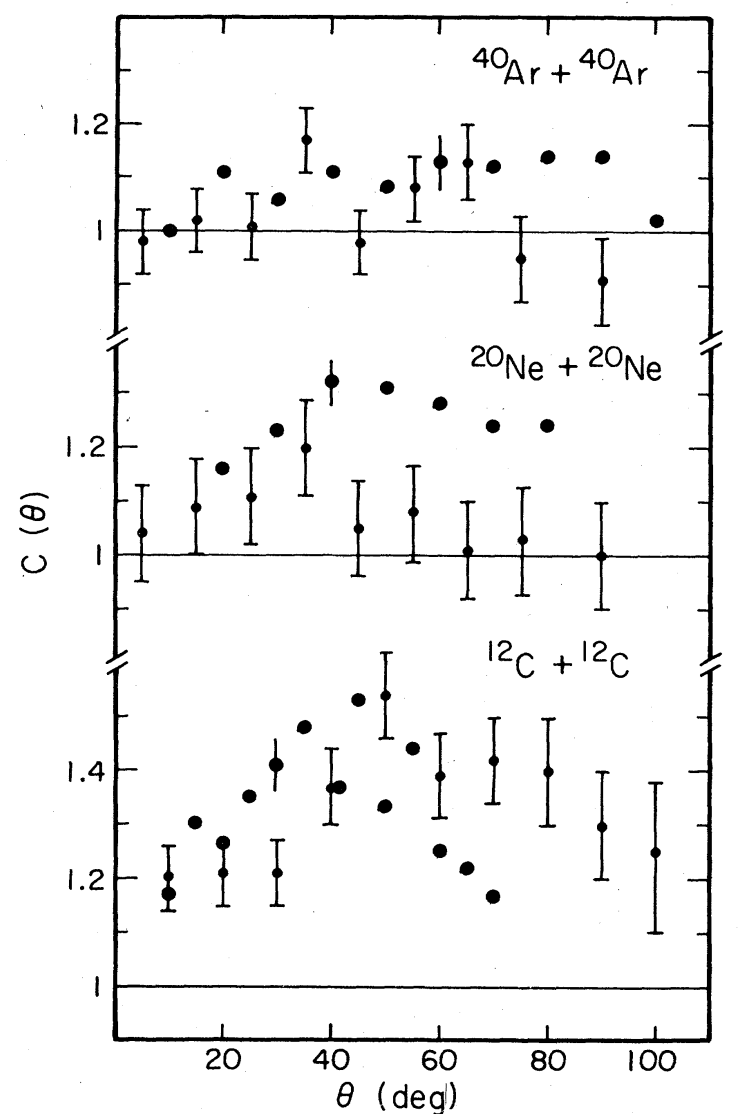

FIG. 10. Variation of the correlation coefficient with the polar angle of the telescope $T$ (see text). The calculation is indicated by the small dots. The heavy dots are the experimental data from Ref. 24. The incident kinetic energy is $800 \mathrm{MeV}$ per nucleon. However, the ${ }^{20} \mathrm{Ne}+{ }^{20} \mathrm{Ne}$ data actually refer to the $\mathrm{Ne}+\mathrm{NaF}$ system at $400 \mathrm{MeV}$ per nucleon (see text).

diminishes their magnitude. As a matter of fact, the data of Fig. 12 is remarkably fitted by the calculation of Hatch and Koonin (see Ref. 8).

In a multiple scattering model such as ours, instead of defining knockout and thermal process, it is more convenient to split the inclusive cross sections into several contributions $\left(\sigma_{n}\right)$, each of which correspond to nucleons having undergone a definite number of collisions $(n)$. It is natural to identify $\sigma_{1}$ with the knockout process. However, in our opinion, it would be misleading to compare $\sigma_{1}$ with the results of Ref. 8. Indeed, taking a large tail in the nucleon momentum distribution is partly equivalent to assuming that the nucleons make two collisions and that the first one has distorted the Fermi spheres attached to the nuclei. On the other hand, the thermal process is not defined unambiguously either. Strictly speaking, no nucleon makes an infinite number of collisions. 


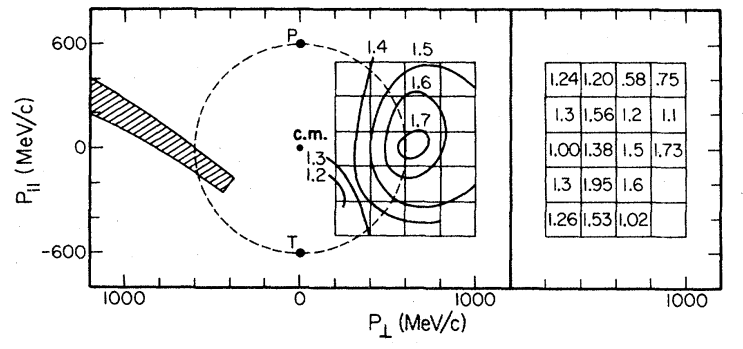

FIG. 11. Variation of the two-nucleon correlation coefficient with $p_{\|}$and $p_{1}$, the c.m. components of the momentum of the proton detected in the telescope $T$ (see text). The system is ${ }^{12} \mathrm{C}+{ }^{12} \mathrm{C}$ at $800 \mathrm{MeV}$ per nucleon in the laboratory system. The $\operatorname{dots} P$ and $T$ indicate the momentum (per particle) components of projectile and target, respectively, in the c.m. system. The shaded area indicates the domain covered by counter $R$ (see text). The experimental data appear as contour plots in the left part of the figure. The results of the calculation are the numbers on the right side of the figure and correspond to the boxes on the left side. The uncertainty in the calculation runs from 0.10 in the upper left corner to 0.40 for the boxes in the lower right corner.

One can define the thermal process contribution as the sum of the $\sigma_{n}$ 's, with $n$ larger than a given value $N$, with of course, some arbitrariness in choosing the value of $N$. In the following, we will try to determine how our calculation distinguishes between the knockout and the thermal process. We will choose $\sigma_{1}+\sigma_{2}$ as the knockout or direct process contribution and define the thermal process contribution as the rest of the inclusive cross sections. This seems to us to be a reasonable

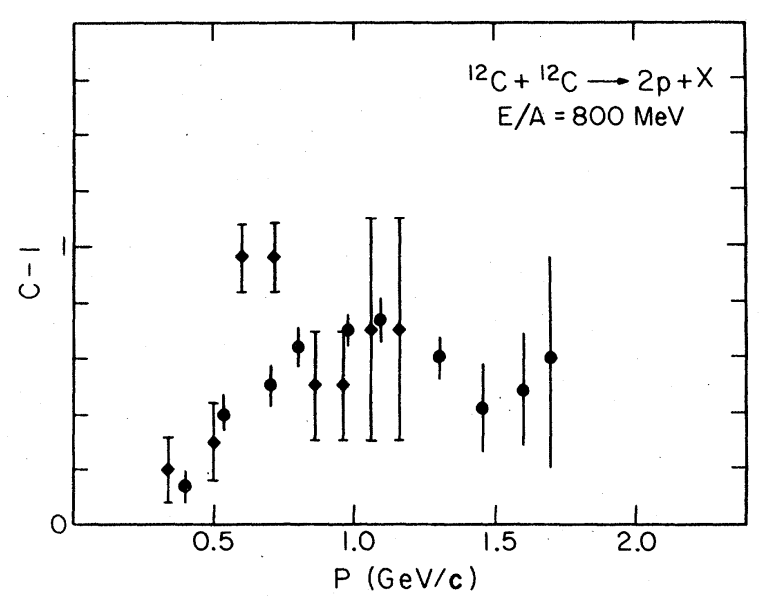

FIG. 12. Same quantity as in Fig. 11, for $\theta_{1 a b}=40^{\circ}$ as a function of nucleon laboratory momentum. This figure corresponds to a cut in the plane of Fig. 11 along a curve starting from $T$ and passing near the experimental peak.

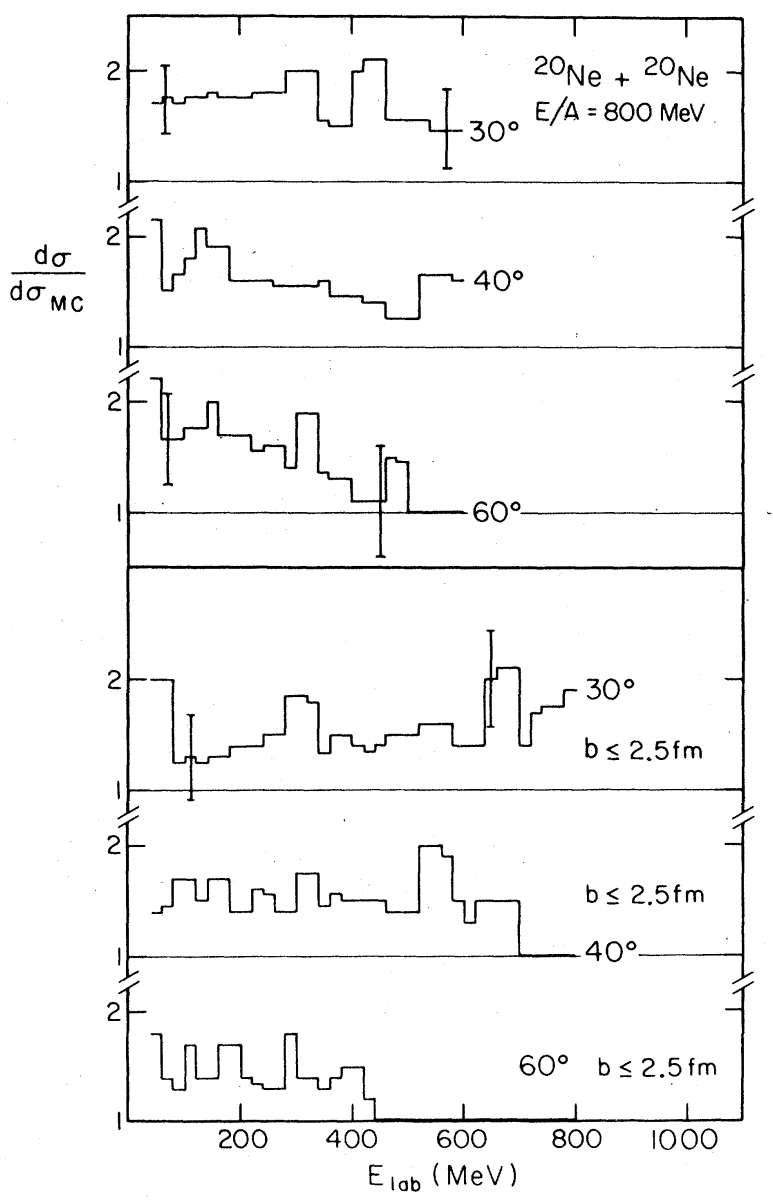

FIG. 13. Plot of the ratio of the calculated inclusive proton cross section to the cross section for protons having made three or more collisions. The system is ${ }^{20} \mathrm{Ne}+{ }^{20} \mathrm{Ne}$ at $800 \mathrm{MeV}$ per nucleon. The lower part of the figure gives the same ratio for impact parameters $\leqslant 2.5 \mathrm{fm}$ (twice the ${ }^{20} \mathrm{Ne}$ radius is $6.08 \mathrm{fm}$ ).

definition, but we stress once again that it contains a certain amount of arbitrariness.

In Figs. 13 through 15, we consider the ratio $R=d \sigma / d \sigma_{M c}$ of the total inclusive cross section to the one obtained by summing the nucleons which have made three or more collisions. We try to extract the gross variation of $R$, with angle, energy, and impact parameter. The excess of $R$ above unity is a measure of the direct process.

In Fig. 13 (upper part), we consider the ${ }^{20} \mathrm{Ne}$ $+{ }^{20} \mathrm{Ne}$ system. The quantity $R$ takes a value around 1.6 at low energy, fairly independent of the angle (we did not plot $R$ for backward angles because of the poor statistics there). But $R$ decrease with energy at a rate which becomes greater as the angle increases. It thus seems difficult for the direct process to produce high energy protons at large angles. It is interesting to look at the values 




FIG. 14. Same quantity as in Fig. 13 for the ${ }^{12} \mathrm{C}+{ }^{12} \mathrm{C}$ system.

of $R$ for central collisions (lower part of Fig. 13). On the average, $R$ is slightly smaller than for all collisions, lying around 1.4 , which is still a rather high value. Moreover, the energy dependence of $R$ at small angles is reversed. This can be viewed as an indication of a shadowing effect. Indeed, a proton which has just made a collision in the c. $m$. region has to pass through the target in order to emerge at $30^{\circ}$, contrary to what happens for a peripheral collision. It has more chance to do that without a further binary collision if it has a higher energy, since the total cross section is smaller and the nucleon is less affected by the agitation of the target nucleons.

Figure 14 contains the results of the calculation of $R$ for the ${ }^{12} \mathrm{C}+{ }^{12} \mathrm{C}$ system. As expected, $R$ reaches higher values, being of the order of 2. A shadowing effect still shows at forward angles and $R$ still goes to unity as energy increases at larger angles. Figure 15 gives a comparison between the ${ }^{12} \mathrm{C}+{ }^{12} \mathrm{C}$ and ${ }^{20} \mathrm{Ne}+{ }^{20} \mathrm{Ne}$ systems for $90^{\circ} \mathrm{c} . \mathrm{m}$. We see that $R$ reaches its limiting value at a kinetic energy of $400 \mathrm{MeV}$.

To summarize, our calculation shows that the direct process contributes about $40 \%$ in the case of the $\mathrm{Ne}+\mathrm{Ne}$ system ( $50 \%$ in the $\mathrm{C}+\mathrm{C}$ system)

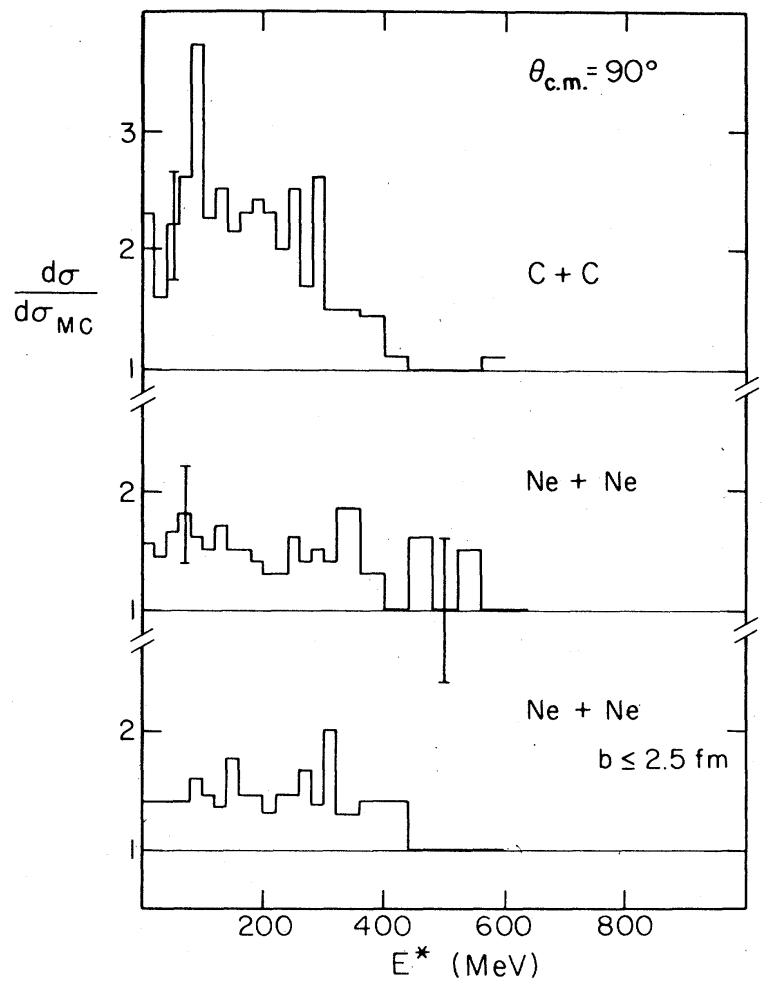

FIG. 15. Same quantity as in Fig. 13 at $90^{\circ} \mathrm{c.m}$. for the ${ }^{12} \mathrm{C}+{ }^{12} \mathrm{C}$ and the ${ }^{20} \mathrm{Ne}+{ }^{20} \mathrm{Ne}$ systems.

at low energies. When the energy of the detected particle increases, the direct process contribution increases at small angles and decreases at larger angles. For central collisions, the direct process contributes less as a whole. We would like to stress that increasing the bombarding energy beyond $800 \mathrm{MeV} / A$ would not necessarily yield an increase of $R$ since the nucleon-nucleon collisions become highly inelastic and a large number of $\Delta$ particles can be produced. The nucleons from their decay have less kinetic energy and thus cannot escape easily from the system. The number of collisions is not reduced and can even be increased. These aspects are studied in Ref. 14 .

\section{DISCUSSION AND CONCLUSION}

We have described a model for the interaction of two nuclei at high energy, which is pictured essentially as a sequence of baryon-baryon collisions treated in a classical relativistic manner. We have compared the predictions of the model with experimental data. We successfully reproduced the proton inclusive cross sections in the ${ }^{12} \mathrm{C}+{ }^{12} \mathrm{C},{ }^{20} \mathrm{Ne}+{ }^{20} \mathrm{Ne}$, and ${ }^{40} \mathrm{Ar}+{ }^{40} \mathrm{Ar}$ systems at 800 $\mathrm{MeV} / A$. The pion data are reproduced fairly well 
on the whole, but the model fails to give the right yield at small momenta in the c. m. system. The two-nucleon correlation data are reproduced very satisfactorily by our model. We stress that all agreement with experiment is obtained without the help of any adjustable parameters. The model does rely on certain assumptions, some of which can be questioned, but calculations have been performed without varying any parameters.

The very success of the model in predicting correctly a broad collection of data strongly suggests that the nucleus-nucleus interaction process is largely dominated by binary, classical, on-shell nucleon-nucleon collisions. Quantum effects (offshell scattering, mean field), three-body (or more) collisions, and exotic phenomena, if any, are therefore expected to be very difficult to detect in the observables investigated in this work. This could no longer be the case for more exclusive quantities. In the multiple scattering process, we have found that the direct or knockout process can contribute for 40 to $50 \%$ on the average. However, this value can change with energy and angle. In particular, high energy protons at large angles can seemingly be produced by multiple scattering only.

One disadvantage of Monte Carlo calculations is that one does not have very much insight into the way a specific assumption affects the final results.
The structures found in the calculated two proton correlation coefficient provides a typical example. Another illustration is provided by density effects. In our model, at least, for small impact parameters, the system undergoes a compression-decompression phase which does not seem to leave any particular trace in the inclusive observables. The detection of such a phenomenon would probably require a multiple particle correlation measurement and a selection of central collision events.

The most questionable assumption of our model, in light of our results, is the introduction of $\Delta$ particles with a definite mass. We are currently investigating this point.

\section{ACKNOWLEDGMENTS}

We are grateful to Dr. S. E. Koonin for many suggestions and a careful reading of the manuscript; to Dr. J. R. Nix and Dr. J. Randrup for useful discussions. We have benefited from the encouragement of T. Mizutani and J. Vandermeulen. We wish also to thank the members of the Kellogg Radiation Laboratory of the California Institute of Technology for their kind hospitality. This work is supported in part by the National Science Foundation Contract No. PHY76-83685.
*On leave of absence from Physics Department, University of Liége, B-4000 Sart-Tilman, Belgium.

${ }^{1}$ G. F. Chapline, M. H. Johnson, E. Teller, and M. S. Weiss, Phys. Rev. D 8 , 4302 (1973).

${ }^{2}$ A. B. Migdal, Zh. Eksp. Teor. Fiz. 61, 2210 (1971) [Sov. Phys.-JETP 34, 1182 (1972)].

${ }^{3}$ T. D. Lee and G. C. Wick, Phys. Rev. D $\underline{4}, 1601$ (1971).

${ }^{4}$ G. D. Westfall, J. Gosset, P. J. Johansen, A. M. Pos-

kanzer, W. G. Meyer, H. H. Gutbrod, A. Sandoval, and

R. Stock, Phys. Rev. Lett. 37, 1202 (1976).

${ }^{5}$ J. Gosset, J. I. Kapusta, and G. D. Westfall, Phys. Rev. C 18,844 (1978).

${ }^{6}$ A. A. Amsden, J. N. Ginocchio, F. H. Harlow, J. R. Nix, M. Danos, E. C. Halbert, and R. K. Smith, Phys. Rev. Lett. 38, 1055 (1977).

${ }^{7}$ A. A. Amsden, F. H. Harlow, and J. R. Nix, Phys. Rev. C 15,2059 (1977).

${ }^{8}$ R. L. Hatch and S. E. Koonin, Phys. Lett. $81 \mathrm{~B}, 1$ (1979).

${ }^{9}$ A. R. Bodmer and C. N. Panos, Phys. Rev. C $\underline{15}, 1342$ (1977); L. Wilets, E. M. Henley, M. Draft, and A. D. Mackellar, Nucl. Phys. A282, 341 (1977).

${ }^{10} \mathrm{~J}$. Hüfner and J. Knoll, Nucl. Phys. A290, 460 (1977);

J. Randrup, ibid. A306, 509 (1979).

${ }^{11}$ R. K. Smith and M. Danos (unpublished).
${ }^{12}$ Y. Yariv and Z. Fraenkel, Phys. Rev. C 20, 2227 (1979).

${ }^{13}$ This feature violates the Lorentz invariance of our calculation. Lorentz invariance properties of our Monte Carlo calculation are discussed in more detail in Ref. 14.

${ }^{14} \mathrm{~J}$. Cugnon, T. Mizutani, and J. Vandermeulen (unpublished).

${ }^{15}$ M. Hirata, J. H. Koch, F. Lenz, and E. J. Moniz, Phys. Lett. 70B, 281 (1977).

${ }^{16}$ M. Hirata, F. Lenz, and Y. Yazaki, Ann. Phys. (N.Y.) 108,116 (1977).

$1 \sqrt{J}$. Cugnon, T. Mizutani, and J. Vandermeulen (unpublished).

${ }^{18}$ Particle Data Group, $N N$ and $N D$ Interactions (above $0.5 \mathrm{GeV} / c$ ). A compilation (Report No. UCRL-20000 $N N, 1970$ ).

${ }^{19}$ G. J. Igo, Rev. Mod. Phys. $\underline{50}, 523$ (1978).

${ }^{20}$ S. Nagamiya et al., Phys. Lett. 81B, 147 (1979); S. Nagamiya et al., Proceedings of the International Conference on Nuclear Structure, Tokyo, 1977, edited by T. Marumori (Physical Society of Japan, Tokyo, 1978); J. Phys. Soc. Jpn. Suppl. 44 378 (1978).

${ }^{21} \mathrm{~S}$. Nagamiya (unpublished).

${ }^{22} \mathrm{~J}$. Carroll, Proceedings of the 4th High Energy Heavy 
Ion Summer Study, Berkeley, 1978, Report No. LBL7766, p. 157 (unpublished).

${ }^{23} \mathrm{R}$. Sandoval et al., Report No. LBL-8771, 1979.

${ }^{24}$ S. Nagamiya, Proceedings of the 4th High Energy Heavy Ion Summer Study, Berkeley, 1978, Report No.
LBL-7766, p. 71 (unpublished).

${ }^{25} \mathrm{H}$. H. Gutbrod, Proceedings of the 4th High Energy Heavy Ion Summer Study, Berkeley, 1978, Report No. LBL-7766, p. 1 (unpublished). 